\title{
The Significance and Evolution of the Stellar Initial Mass Function
}

\author{
Ethan Kuperman \\ Horace Greeley High School, 70 Roaring Brook Road, Chappaqua, New York, 10514, USA; ethanckuperman@gmail.com
}

\begin{abstract}
It remains undisputed in the current star formation paradigm that the structure and evolution of a star are primarily dependent on its mass. The initial mass function (IMF), the probability distribution function of stellar masses at birth, is consequently a vital function to stellar astrophysics. Since its creation in 1955 by Edwin Salpeter, the IMF has undergone much study and modification, but a definite understanding of the origin and form of the stellar IMF remains elusive. This review will consider the contributions of a multitude of astrophysicists to the study of the IMF in a comprehensive overview and pose several avenues for future research.

KEYWORDS: Physics and Astronomy; Astronomy and Cosmology; Theory; Star Formation; Initial Mass Function.
\end{abstract}

\section{Introduction}

Stars have long fascinated humans on Earth. Luminous spheroids of plasma formed from vast clouds of cosmic dust and gas, they captivate us as they shine high on the celestial sphere. We have gone a long way since the first study of stars by Galileo Galilei in 1610, using ever-developing technologies and challenging ourselves to advance the cogent components of theories for star formation - the most integral of which, arguably, is the initial mass function. Stars of varying masses form from interstellar gas and dust, and it is necessary to interpret these masses to understand the characteristics of foreign galaxies.

The IMF, which is based on the initial mass of a star, is a probability distribution which can be used to describe the distribution of stellar masses at birth. The distribution function, fit to a histogram indicating the frequency of stars for mass intervals ranging from $m$ to $m+\Delta m$, is an operational construct defined as either a power law (also known as the Pareto distribution) or a log-normal distribution, the two top contenders for a best-fitting function. Each function has vastly different implications; a power law distributes masses such that any distribution from a given sample will be the same regardless of the size of its progenitor cloud. Contrastingly, the log-normal form is a result of multiplicative processes and implies that several factors contribute to the outcome. ${ }^{1}$ As evident from the conclusions drawn intrinsically by each form, the IMF reveals information about the star formation rate of the universe (SFR) and the physical processes which cause star formation and result in a variety of stellar properties by designating the fraction of stars at different masses. ${ }^{2}$ Beyond just measuring star counts and suggesting properties, the IMF is also useful in inferring galaxy characteristics and is often taken into consideration for star formation modeling. ${ }^{3}$

Past reviews of the IMF and the functional forms that define it cover in great depth its importance and the general course of development, but these reviews are often quite complex by nature. This review aims to discuss the significance and evolution of the IMF in a clear and succinct manner to highlight its influence in the field of astrophysics. Furthermore, complications with current methods of inferring and representing the IMF and future directions will be established.

\section{- Discussion}

\section{Salpeter and The First IMF :}

Since its founding by Austrian astrophysicist Edwin Salpeter, the initial mass function has established itself as a vital component of modern astrophysics. The stellar IMF is closely tied to the star formation rate (SFR), and both are necessary for studies of observed properties and chemical abundances of foreign galaxies. Colors and spectra of groups of stars primarily depend on the SFR and stellar IMF, which are used to interpret various properties based on mass. The publication of Salpeter's 1955 paper, The Luminosity Function and Stellar Evolution, gave the definition of the first stellar IMF of the canonical shape $\xi(\mathrm{m})=\mathrm{d} N / \mathrm{dm}$ ), with $\mathrm{d} N$ representing the number of locally formed stars with masses ranging from $m$ to $m+\Delta m$. The Salpeter IMF is also represented as $\xi(\log m) \approx A(m$ $\left(M_{0}\right)^{-\mathrm{x}}$, a relation with a suggested index of $\mathrm{x}=1.35$, limited to the solar neighborhood where $\xi(m)$ is a smooth function of the input $\mathrm{m}$. Salpeter's IMF described the mass distribution for a population of stars of masses between 0.4 and 10 solar masses at birth on the main sequence, but grew uncertain as inputs fell below one solar mass as it is difficult to detect the dim, overlapping population of low-mass stars. As observational equipment and techniques improved, the Salpeter IMF was eventually considered to flatten below one solar mass. ${ }^{4}$ Similarly, massive stars are impossible to fully account for in any observationally determined IMF due to their short lifetimes.

Due to the natural phenomenon of stars moving from the main sequence and becoming red giants and eventually white dwarfs, black holes, and neutron stars as they reach the end of their lifetimes, they must be drawn back to their original positions on the main sequence to infer the IMF. The IMF is a restricted version of a distribution of present-day masses 
of stars (the Present-Day Mass Function or PDMF) based on the Luminosity Function, determined by the parameterized luminosity-mass relation $L=M^{x}$ where $x$ ranges from 1 to 6 and is commonly 3.5 for main-sequence stars. By using the PDMF as a basis for IMF development, only minor constraints must be made to develop the related function for initial mass.

Essentially, the IMF is represented by a mass distribution of stars in a selected area and time, created by designating mass ranges to a continuous set of histogram bins (Figure 1). A time interval can be designated for the purpose of understanding the birth of a sample of stars or for a region over its lifetime. This distribution can signify multiple important properties based on an inference of characteristics due to mass, including the luminosities and lifetimes of stars as well as the contingent energy feedback due to supernovae, which can assist researchers in forming a general picture of the region being studied. When graphed, the power law form of the IMF is commonly placed upon $\log -\log$ axes such that the intrinsic curve is eliminated, and the function has a linear shape despite its exponential nature on normalized axes.

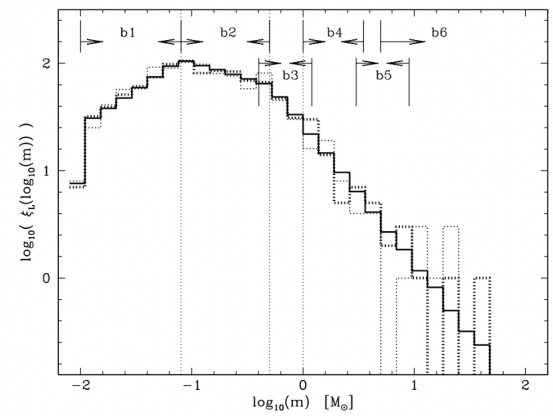

Figure 1: A histogram IMF for $10^{6}$ stars, with thick and thin dotted indicators for two versions of $10^{3}$ star plots. Vertical dotted lines indicate masses where the power law exponent changes, suggesting variation. From Kroupa, 2001. ${ }^{12}$

\section{The Development of the gIMF and cIMF by Integration of} IMFs:

Just as star formation exists on scales both small and large, IMFs exist for every range of star forming regions. The galaxy-wide IMF (gIMF) is the summation of IMFs over a galaxy, synonymous with the probability distribution function over said galaxy. In the integrated galaxy theory (IGIMF) theory developed by Pavel Kroupa, the gIMF is calculated by integration over all clusters formed over an epoch of $10 \mathrm{Myr}$ within the galaxy in which the gIMF is applicable.

As mentioned above, but discussed in more depth presently, the shape of the gIMF does not necessarily match the shape of the IMF because low-mass embedded star clusters are implied not to have massive stars. ${ }^{5}$ Specifically, it is insinuated that the gIMF has a steep exponent greater than or equal to 2.8 and does not follow the Salpeter power-law. ${ }^{6}$ The gIMF, however, is similar to the IMF in a galaxy with a star formation rate of approximately one solar mass per year and solar metallicity. The gIMF proposed by the IGIMF varies with the star formation rate and metallicity of its galaxy because, on a smaller scale, the IMF varies among stars formed from their progenitor molecular clouds. Recently, an important forward step has been taken in the study of the gIMF - the recently developed Python module, GalIMF, is able to calculate the gIMF following the IGIMF theory based on the galaxy-wide star formation rate and metallicity. By synthesizing stellar populations for galaxies, the GalIMF module drives the construction of galactic star formation history empirically. ${ }^{7}$

The cosmic IMF (cIMF, not to be confused with the CIMF, the IMF for star clusters) is similarly the integration of all smaller-scale IMFs. Presently, no definite procedure exists to define the cIMF in entirety, but it is implied that star populations within galaxies must be self-consistent to obtain a cIMF. ${ }^{8}$ This is simply due to the problematic nature of widespread variation if it is confirmed to exist (Figure 2).

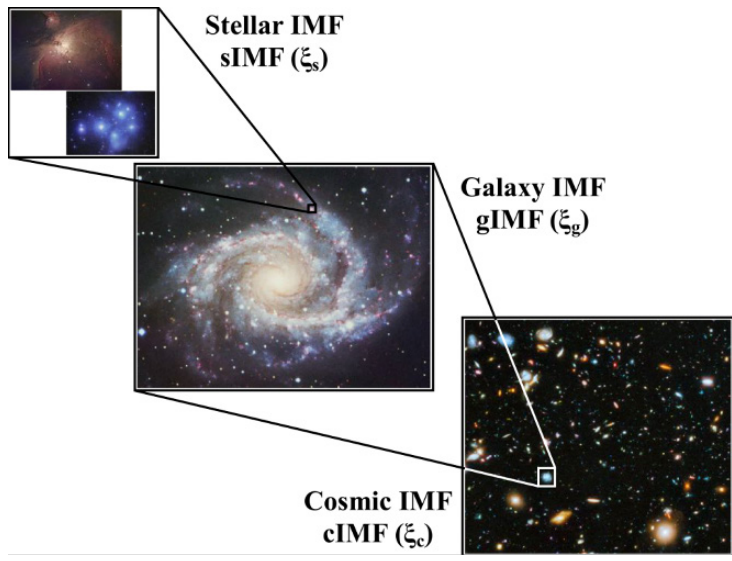

Figure 2: A diagram of regions with theoretically calculable IMFs ranging from the smallest scale (stellar) to the largest (cosmic). Variation within the stellar IMF will affect the universality of the gIMF and, consequently, the same will occur from the gIMF to the cIMF. From Hopkins, 2020.4

\section{Inferring the IMF :}

The IMF cannot be entirely derived from observations nor is it a singular equation; rather, it is purely a statistical function which varies based on mass input. Therefore, it is an operational construct to characterize galaxies and star formation. In actuality, the clusters necessary for a complete observation of the IMF are either too distant or not massive enough to be observed, with ejections and supernovae further complicating full-scale inferences. ${ }^{9}$ The output varies based on the inferring method used and the characteristics of the region to which it is applied as well as error in measurement. Galaxies forming stars at a rate higher than average tend to have an abundance of massive stars, indicated by a peak further to the right on the $\mathrm{x}$-axis of an IMF histogram. ${ }^{10}$ Oppositely, passive galaxies which tend to have excess low-mass stars have a peak further to the left on the $\mathrm{x}$-axis. ${ }^{11}$ Many ways to infer the IMF exist, all of which have differing methodologies and useful applications. The most essential of these methods will be discussed presently.

The photometric approach determines an IMF through broadband photometry, deriving masses from the mass-luminosity relationship of stars. This method, while popular for usage for individual stars and systems, is uncertain for massive stars. 
The spectroscopic approach, a solution to achieving higher accuracy for massive stars, places coordinates on a Hertzsprung-Russell diagram for an $\mathrm{x}$-axis of temperature and spectral class and a $\mathrm{y}$-axis of luminosity, often measured in Kelvin and solar luminosities respectively. An IMF can be inferred through analyzing the dependence of a star's evolutionary properties and therefore stage on the Hertzsprung-Russell diagram on its initial mass.

The integrated light approach infers an IMF and SFR from the strength of $\mathrm{Ha}$ lines, the brightest emission lines in the visible range, and several colors which determine the characteristics of the region being studied. ${ }^{12}$

The chemical abundance approach focuses on the outcome of supernovae, which enriches the stellar populations influenced by said supernovae and can be used to trace IMFs based on metallicity. ${ }^{13}$

Notably, inferring the IMF for a star cluster is far easier than for field stars due to the nature of stars being born in one cluster at the same time, causing a simpler process of constructing a histogram from their masses at birth.

The Continuous Development of the Salpeter IMF and its Universality:

Whether the IMF is universal or variable has persisted as an essential question for astrophysicists. To better understand the universality of the IMF, various researchers have investigated alternate methods to develop the function. Such efforts included the study of stellar nucleosynthesis and the resulting chemical evolution and UV luminosities which are now more routinely used to trace the intermediate-mass stellar population. ${ }^{14}$ In a relatively recent development, the most successful of these alternate methods is gleaned from gravitational lensing, which led to the creation of the IMF mismatch $\alpha \equiv M L D E$ in $/ M S P S E$ in , the ratio of stellar mass determined by gravitational lensing to that determined by stellar population synthesis models. This ratio agreed with the Salpeter IMF in a reinforcement of its validity. ${ }^{15} \mathrm{~A}$ key result of the application of gravitational lensing to the IMF is the implication that the IMF mismatch increases with the velocity dispersion of a galaxy and implies a non-universal IMF.

Additionally, and also in support of a variable IMF, the application of parameter correction from systematic bias led to discovery of some variation which suggested that the IMF was radically different among the ancient star population. ${ }^{16}$ And, as an integration of varying IMFs, the Milky Way gIMF implies both spatial and temporal variability. On the strength of these studies, researchers are presently advocating for a non-universal IMF and, consequently, gIMF as the basis for further studies. This is a step in the direction of a substantial paradigm shift, as the IMF has previously been considered to be universal.

The validity of the Salpeter IMF has also been considered by astrophysicists, as the field of stellar astrophysics has grown vastly since its proposal. For instance, Salpeter's suggestion for the usage of the index 1.35 was disputed due to the fact that the Salpeter IMF was based on the accepted Galaxy disk age at the time, 6 Gyr, which we now know to be approximately 12 Gyr. The index was corrected and instead suggested to be $1.05{ }^{17}$ This correction was itself disputed for stars larger than eight solar masses due to a prior observational effort using spectroscopy where 1.35 was determined to be the correct value for massive stars. ${ }^{18}$ Presently, the usage of a log-normal functional form and (more commonly) a parameter-based power-law offer irrefutably more accurate IMFs.

Contemporary Log-Normal and Power-Law IMF Forms:

The result of the many aforementioned developments is the surfacing of major advances in understanding and developing new forms of the IMF (Figure 3). During a period where the IMF was considered log-normal-like, the following function form was created by Glenn Miller and John Scalo. ${ }^{19}$

$$
\log _{10} \xi\left(\log _{10} m\right)=A-\frac{1}{2}\left(\log _{10} \sigma\right)^{2}\left(\log _{10} \frac{m}{m_{0}}\right)^{2}
$$

Gilles Chabrier introduced another log-normal form which is modifiable with variable parameters applied to both individual stars and systems with masses less than one. Chabrier also restated the power-law form for masses greater than one. ${ }^{20}$ The variables $a, b$, and $c$ are used in this review for convenience. This form offered the first jump from Salpeter's original smooth function to a variable IMF.

$$
\xi(m)=a\left(\frac{1}{\ln 10 m}\right) \exp \left[\frac{(-\log m-\log b)^{2}}{\left(2 c^{2}\right)}\right]
$$

For individual stars, $\mathrm{a}=0.158, \mathrm{~b}=0.08$, and $\mathrm{c}=0.69$. For binary and multiple systems, $\mathrm{a}=0.086, \mathrm{~b}=0.22$, and $\mathrm{c}=0.57$.

$$
\xi(m)=k m^{2.3 \pm 0.3}
$$

Also intrinsic are Pavel Kroupa's piecewise parameters for mass ranges for the broken power-law form of the Salpeter IMF for the range $m$ to $m+\Delta m .^{21,22}$ Prior to the introduction of this piecewise form, the IMF was a subject of much debate as the topic of universality versus variability grew in popularity. As evidence for variation emerged from improved methods of inferring the IMF, the need for more accurate parameters grew until the following were developed.

$$
\begin{gathered}
\xi(m) d m \propto m^{\alpha} d m \\
\xi(m)=0.26 m^{-0.3} \text { for } 0.01 \leq m<0.08 \\
\xi(m)=0.035 m^{-1.3} \text { for } 0.08 \leq m<0.5 \\
\xi(m)=0.019 m^{-2.3} \text { for } 0.5 \leq m<\infty
\end{gathered}
$$

These parameters offer the most accurate way of empirically estimating the power-law IMF; therefore, the Kroupa IMF is considered to be the standard modern function. Exponents are fairly uncertain at less than 0.5 solar masses. Kroupa's IMF is accepted because it is statistically corrected for systems too far to detect through spectroscopy and too close to be resolved. Prior IMFs which did not account for these errors typically overestimated masses of stars and therefore underestimated their densities. The IMF, previously assumed to be an invariant probability density function, is not commonly known as a parameterized distribution to which Kroupa's power law applies. $^{23}$ 


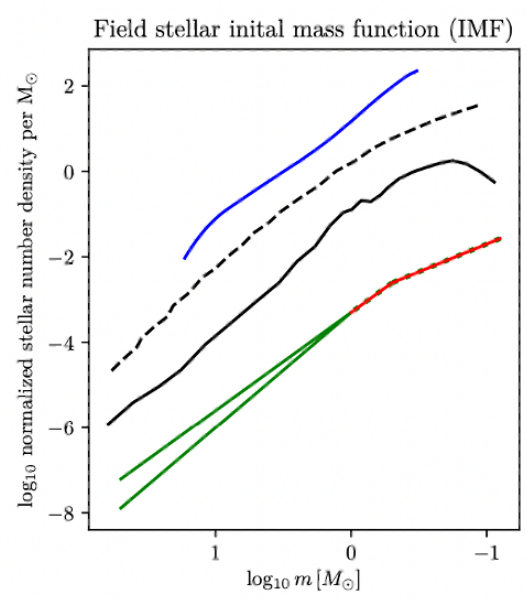

Figure 3: The Salpeter IMF in blue, the Scalo IMF in solid black, the Miller and Scalo IMF in dashed black, the Kroupa IMF in red, and the Scalo IMF as the bottom piece of the Kroupa IMF divergence in green. The Massey IMF for non-field stars, taken from spectroscopy, is the upper piece of the divergence (also in green). From Kroupa, 2019.25

\section{Conclusion}

Today, the contributions of astrophysicists past and present leave a substantial variety of developments in IMF research, but there are also many unfilled gaps in the current paradigm. The creation of various forms has offered a diverse number of options for stellar astrophysicists, and growth in the usage of modules and data analysis code bodes well for future studies of the IMF. However, ambiguity is commonplace amongst histograms developed using varying methods for different sample sizes and regions, with upper bins often being dropped for low sample sizes, clearly indicating the incompleteness of census data available. As the IMF increasingly shows signs of being variable through census data, it is imperative to leave behind tendencies towards universality and consider parameterized forms and additional distributions.

The upcoming launch of the James Webb Space Telescope offers an incredible opportunity to further investigate star formation by exploring high-redshift systems forming stars, much like the Gaia photometry and parallax mission cataloged census data for over one billion stars in an unprecedentedly complete survey. ${ }^{24}$ Astrophysicists are hopeful that the information acquired from such a study will offer constraints to the variation of the IMF as well as investigations into its universality. ${ }^{25} \mathrm{~A}$ heightened understanding of turbulence and the structure of stars will also provide opportunities to advance the development of the IMF and other frontiers of stellar astrophysics. $^{26,27}$

\section{- References}

1. J. Kirkpatrick, C. Gelino, J. Faherty, A. Meisner, D. Caselden, A. Schneider, F. Marocco, A. Cayago, D.Smart, P. Eisenhardt, M. Kuchner, E. Wright, M. Cushing, K. Allers, D. Gagliuffi, A. Burgasser, J. Gagne, S. Logsdon, E. Martin, J. Ingalls, P. Lowrance, E. Abrahams, C. Aganze, R. Gerasimov, E. Gonzales, C. Hsu, N. Andersen, P. Beaulieu, G. Colin, C. Elachi, S. Goodman, L. Gramaize, L. Hamlet, J. Hong, A. Jonkeren, M. Khalil, D. Martin, W. Pendrill, B. Pumphrey, A. Rothermich, A. Sainio, A. Stenner, C. Tenner, M. Thevenot, N. Voloshin, J. Walla, Z. Wedracki. "The Field Substellar Mass Function Based on the Full-sky 20 pc Census of 525 L, T, and Y Dwarfs." The
Astrophysical Journal Supplement Series. 235, 7 (2021).

2. T.Jerabkova. "Stellar populations in gravitationally bound systems." The Repository of the University of Bonn. (2020).

3. J. Scalo. "Five Years of IMF Variation: The Intermediate-Mass Stars.”The Initial Mass Function 50 Years Later. 1, 23 (2005)

4. J. Scalo. "The Stellar Initial Mass Function." Fundamentals of Cosmic Physics. 11, 1 (1986).

5. Z. Yan, T. Jerabkova, P. Kroupa. "The optimally sampled galaxywide stellar initial mass function."Astronomy and Astrophysics. 607, A126 (2017).

6. P. Kroupa \& C. Weidner. "Galactic-Field Initial Mass Function of Massive Stars.” Astrophysical Journal. 598, 1076 (2003).

7. H. Haghi, V. Amiri, A. Zonoozi, I. Banik, P. Kroupa, M. Haslbauer. "The Star Formation History and Dynamics of the Ultra-diffuse Galaxy Dragonfly 44 in MOND and MOG." Astrophysical Journal Letters. 884, 25. (2019).

8. A. Hopkins, Presentation: "Measuring the Stellar Initial Mass Function." (2020.)

9. B. Elmegreen. "The Stellar Initial Mass Function in 2007: A Year for Discovering Variations." 4th Spitzer Science Center Conference. 4, 14 (2007).

10. M. Gunawardhana, A. Hopkins, R. Sharp, S. Brough, E. Taylor, J. Bland-Hawthorn, C. Maraston, R. Tuffs, C. Popescu, D.

Wijesinghe, D. Jones, S. Croom, E. Sadler, S. Wilkins, S. Driver, J. Liske, P. Norberg, I. Baldry, S. Bamford, J. Loveday, J. Peacock, A. Robotham, D. Zucker, Q. Parker, C. Conselice, E. Cameron, C. Frenk, D. Hill, L. Kelvin, K. Kuijken, B. Madore, B. Nichol, H. Parkinson, K. Pimbblet, M. Prescott, W. Sutherland, D. Thomas, E. van Kampen. "Galaxy And Mass Assembly (GAMA): The star formation rate dependence of the stellar initial mass function." Monthly Notices of the Royal Astronomical Society. 415, 1647 (2011).

11.M. Cappellari, R. McDermid, K. Alatalo, L. Blitz, M. Bois, F. Bournaud, M. Bureau, A. Crocker, R. Davies, T. Davis, P. de Zeeuw, P. Duc, E. Emsellem, S. Khochfar, D. Krajnovic, H. Kuntschner, P. Lablanche, R. Morganti, T. Naab, T. Oosterloo, M. Sarzi, N. Scott, P. Serra, A. Weijmans, L. Young. "A systematic variation of the stellar initial mass function in early-type galaxies." Nature. 484, 485 (2012).

12.R. Kennicutt. "The rate of star formation in normal disk galaxies." Astrophysical Journal. 272, 54 (1983).

13.T. Gutcke \& V. Springel. "Simulating a metallicity-dependent initial mass function: consequences for feedback and chemical abundances." Monthly Notices of the Royal Astronomical Society. 482, 118 (2018).

14.J. Audouze \& B. Tinsley. "Chemical Evolution of Galaxies.” Astronomy and Astrophysics. 14, A76 (1976).

15.T. Treu, M. Auger, L. Koopmans, R. Gavazzi, P. Marshall, A. Bolton. "The initial mass function of early-type galaxies." Astrophysical Journal. 709, 1195 (2009).

16.P. Kroupa. "On the variation of the initial mass function." Monthly Notices of the Royal Astronomical Society. 322, 231 (2001).

17.H. Zinnecker, "Up2010 Introduction: Massive Star Formation and the Upper End of the IMF." Up2010 ASP Conference Series. 440, 3 (2010).

18.P. Kroupa, C. Tout, G. Gilmore. "The distribution of low-mass stars in the Galactic disc." Monthly Notices of the Royal Astronomical Society. 262, 545 (1993)

19.G. Miller \& J. Scalo. "The Initial Mass Function and Stellar Birthrate in the Solar Neighborhood." Astrophysical Journal Supplement Series. 41, 513 (1979).

20.G. Chabrier. "Galactic Stellar and Substellar Initial Mass Function." 
Publications of the Astronomical Society of the Pacific. 115, 763

(2003).

21.P. Kroupa. "The Initial Mass Function of Stars: Evidence for Uniformity in Variable Systems.” Science. 295, 82 (2002).

22.M. Mac Low \& R. Klessen. "Control of star formation by supersonic turbulence.” Reviews of Modern Physics. 76, 125 (2004).

23.T. Jerabkova, A. Zonoozi, P. Kroupa, G. Beccari, Z. Yan, A.

Vazdekis, Z. Zhang. "Impact of metallicity and star formation rate on the time-dependent, galaxy-wide stellar initial mass function." Astronomy and Astrophysics. 620, A39 (2018).

24.A. Sollima. "The stellar initial mass function of the solar neighbourhood revealed by Gaia." Monthly Notices of the Royal Astronomical Society. 489, 2377.

25.T. Jerabkova, P. Kroupa, J. Dabringhausen, M. Hilker, K. Bekki. "The formation of ultra compact dwarf galaxies and massive globular clusters." Astronomy and Astrophysics. 608, A53 (2017).

26.M. Mac Low. “From Gas to Stars Over Cosmic Time.” Science. 340, 6140 (2013)

27.P. Kroupa \& T. Jerabkova. "The Salpeter IMF and its descendants." Nature. 3, 482 (2019).

- Author

Ethan Kuperman is a sophomore at Horace Greeley High School in Chappaqua, New York, studying astrophysics with a focus on star formation and evolution 